\title{
ADROPIN AS A POTENTIAL BIOMARKER OF NUTRITIONAL STATUS AND CARDIAC FUNCTION IN HEMODIALYZED PATIENTS.
}

\author{
Kałużna Małgorzata', Schwermer Krzysztof' ${ }^{2}$, Hoppe Krzysztof ${ }^{2}$, Człapka-Matyasik Magdalena $^{3}$, Sawicka-Gutaj Nadia', \\ Yusuf Ibrahim Aisha ${ }^{2}$, Minczykowski Andrzej ${ }^{4}$, Pawlaczyk Krzysztof ${ }^{1}$, Ziemnicka Katarzyna', Ruchała Marek ${ }^{1}$ \\ 1 Department of Endocrinology, Metabolism and Internal Medicine, Poznan University of Medical Sciences, Poland \\ 2 Department of Nephrology, Transplantology and Internal Diseases, Poznan University of Medical Sciences, Poland \\ ${ }^{3}$ Poznań University of Life Sciences, Department of Human Nutrition and Hygiene, Poznan, Poland \\ ${ }^{4}$ Department of Cardiology - Intensive Therapy, Poznan University of Medical Sciences, Poland
}

Introduction: Adropin, a newly discovered peptide hormone, seems to have broad implications in energy homeostasis and metabolism in humans. Its main role is in maintaining appropriate carbohydrate and lipid economy. However, the aim of this study was to look at the novel role of adropin as a potential marker for cardiac function and nutrition status in hemodialyzed (HD) patients. To the best of our knowledge, this was the first study to consider the relationship between serum adropin concentration and clinical status of HD patients.

Description of methods/design: The study consisted of 41 HD patients (29 males; median age \pm 53 years). Adropin levels along with circulating $N$-terminal pro b-type natriuretic peptide (NT-proBNP), troponin $T$ (cTnT), albumin (Alb), fasting serum insulin and glucose levels, insulin resistance index (HOMA-IR), insulin-like growth factor 1 (IGF-1) and parameters of lipid economy were assessed. Additionally anthropometric measurements were taken and echocardiography was performed in each patient.

Results: Adropin levels were negatively correlated with: body mass $(r=-0.469)$, BMI ( $r=-0.445)$ and triglycerides (TAG) $(r=-0.369)$. Also there was a significant relationship between adropin concentration and IGF-1 $(r=-0.374)$. However adropin levels were not correlated with glucose and insulin levels and HOMA-IR (Table 1). Strong positive correlations were observed between adropin level and $c \operatorname{TnT}(r=0.325)$ as well as NT-proBNP ( $r=0.355)$. Adropin was related to: left ventricular ejection fraction (LVEF) $(r=-0.499)$ and left ventricular systole diameter (LVSd) $(r=0.451) \quad$ (Table 2). No correlation was found between adropin and blood pressure.

Conclusion: Adropin seems to be a negative predictor of nutrition in HD patients. The relationship between adropin and markers of cardiac insufficiency might indicate a new link between myokines and cardiovascular disease (CVD) in dialyzed patients. Adropin could potentially be a new candidate for a marker of cardiac insufficiency in HD patients.

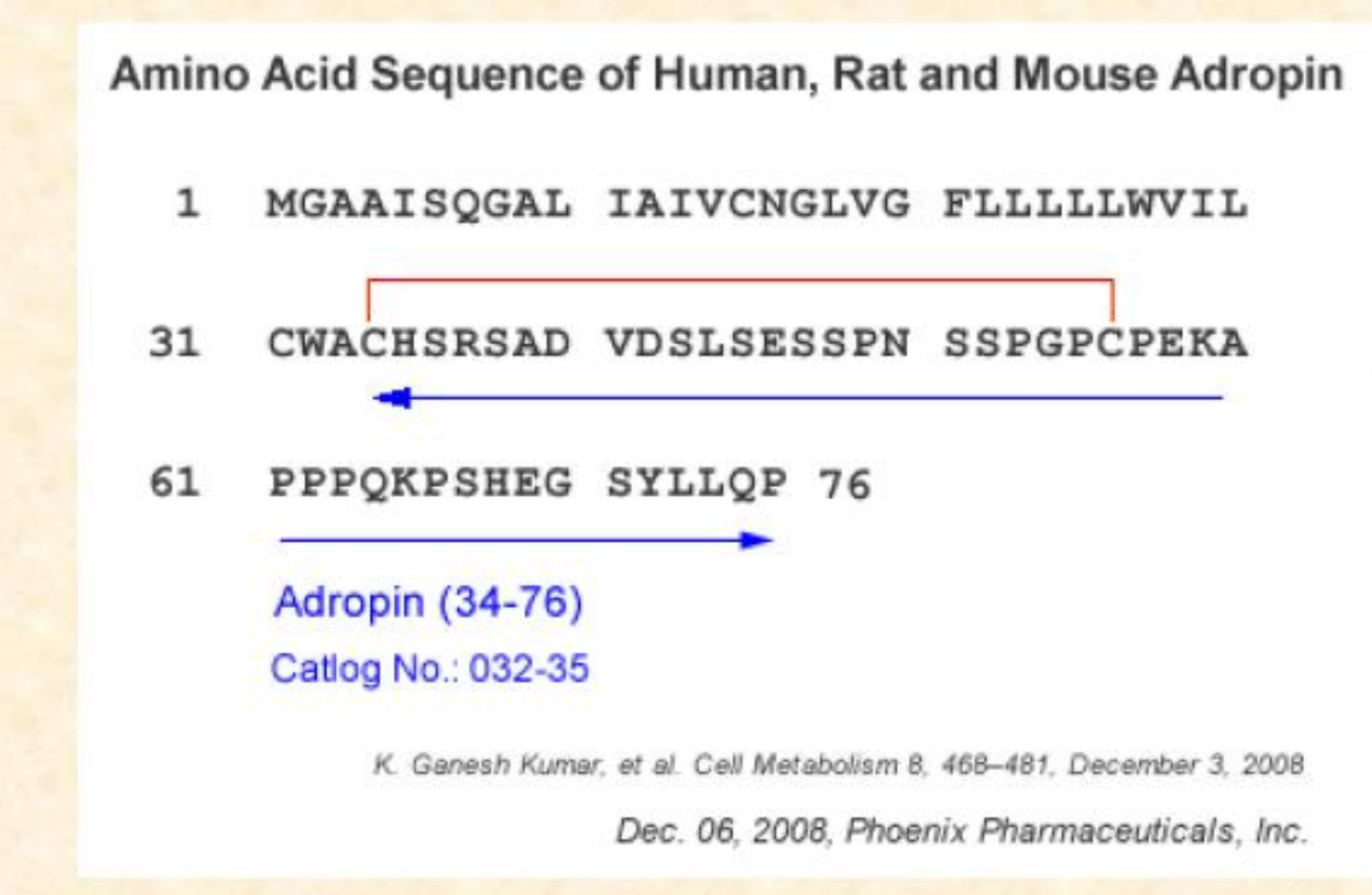

Figure 1.

Adropin structure.

\begin{tabular}{|c|c|}
\hline Parameter & Adropin \\
\hline body mass & $\begin{aligned} \mathrm{r} & =-0.468 \\
\mathrm{p} & =0.037 *\end{aligned}$ \\
\hline BMI & $\begin{array}{l}\mathrm{r}=-0.445 \\
\mathrm{p}=0.049 *\end{array}$ \\
\hline TAG & $\begin{array}{l}\mathrm{r}=-0.369 \\
\mathrm{p}=0.027^{*}\end{array}$ \\
\hline albumin & $\begin{array}{c}\mathrm{r}=-0.22 \\
\mathrm{p}=0.077 *\end{array}$ \\
\hline IGF-1 & $\begin{array}{l}\mathrm{r}=-0.374 \\
\mathrm{p}=0.024 *\end{array}$ \\
\hline insulin & $\begin{array}{c}\mathrm{r}=-0.094 \\
\mathrm{NS}\end{array}$ \\
\hline HOMA-IR & $\begin{array}{c}\mathrm{r}=-0.003 \\
\mathrm{NS}\end{array}$ \\
\hline
\end{tabular}

Table 1.

Correlation coefficient between adropin and laboratory parameters. $*_{p}<0.05$

\begin{tabular}{|c|c|}
\hline Parameter & Adropin \\
\hline cTn-T & $\mathrm{r}=0.325$ \\
& $\mathrm{p}=0.046^{*}$ \\
\hline NT- & $\mathrm{r}=0.355$ \\
proBNP & $\mathrm{p}=0.034^{*}$ \\
\hline LVEF & $\mathrm{r}=-0.499$ \\
& $\mathrm{p}=0.008^{*}$ \\
\hline LVSd & $\mathrm{r}=0.451$ \\
& $\mathrm{p}=0.023^{*}$ \\
\hline
\end{tabular}

Table 2.

Correlation coefficient between adropin and cardiac status biomarkers and $\mathrm{ECHO}$ parameters. $*_{p}<0.05$

\section{Contact:}

Małgorzata Kałużna,MD: kaluznama@gmail.com

Prof. Marek Ruchata, MD, PhD: mruchala@ump.edu.pl 